\title{
Formación del precio de las transacciones internacionales de electricidad entre Colombia y Ecuador
}

Recibido: Abril 19 de 2013 - Aceptado: Febrero 17 de 2014

Doi: dx.doi.org/10.12804/rev.econ.rosario.17.01.2014.03

\author{
John J. García* \\ Universidad EAFIT \\ Catalina Gómez ${ }^{\dagger}$ \\ Universidad EAFIT \\ Santiago Bohórquez ${ }^{\ddagger}$ \\ Universidad EAFIT
}

\section{Resumen}

En este artículo se describe la operación de las transacciones internacionales de electricidad entre Colombia y Ecuador, su principal regulación y las características más importantes de ambos mercados haciendo énfasis en la formación del precio. Además se hacen dos ejercicios econométricos, análisis de componentes principales y beta convergencia, para observar si ha

* Economista, Ph. D en Economía. Profesor Departamento de Economía, Universidad EAFIT, Colombia. AA3300 Medellín (Colombia). Teléfono: (+57 4) 261 9549, Fax: (+57 4) 266 4884. Correo electrónico: jgarcia@eafit.edu.co

+ Economista, Magíster en Economía. Prof. Departamento de Economía, Universidad EAFIT, Colombia. Correo electrónico: cgomezt1@eafit.edu.co

$\ddagger$ Economista. Profesor Departamento de Economía, Universidad EAFIT, Colombia. Correo electrónico: sbohorqu@eafit.edu.co

Para citar este artículo: García, J. J., Gómez, C., \& Bohórquez, S. (2014). Formación del precio de las transacciones internacionales de electricidad entre Colombia y Ecuador. Revista de Economía del Rosario, 17(1), 63-87. doi: dx.doi.org/10.12804/rev.econ.rosario.17.01.2014.03 
existido convergencia en precios entre estos dos países llegando a la conclusión de no convergencia en precios entre los dos mercados.

Clasificación JEL: L11, L14, D43.

Palabras Clave: integración energética, análisis de componentes principales, convergencia en precios, Colombia - Ecuador.

\title{
Price Formation in International Electric Transactions between Colombia and Ecuador
}

\begin{abstract}
This article describes the operation of the international electric transactions between Colombia and Ecuador, its principal regulation and the most important characteristics of both markets paying special attention to the price formation. There is also two econometrics exercises, principal component analysis and beta convergence, to see if there has been price convergence between these two countries showing that there is no price convergence between both markets.

JEL Classification: L11, L14, D43.

Keywords: energetic integration, principal component analysis, price convergence, Colombia - Ecuador.

\section{Formação do preço das transações internacionais de eletricidade entre a Colômbia e o Equador}

\section{Resumo}

Neste artigo descreve-se a operação das transações internacionais de eletricidade entre a Colômbia e o Equador, sua principal regulação e as características mais importantes de ambos os mercados fazendo ênfase na formação do preço. Além disso, realizam-se dois exercícios econométricos, análise de componentes e beta convergência, para observar se tem existido convergência em preços entre estes dois países, concluindo a não convergência em preços entre os dois mercados.

Classificação JEL: L11, L14, D43.

Palavras-chave: integração energética, Análise de componentes principais, Convergência em preços, Colômbia-Equador. 


\section{Introducción}

Colombia y Ecuador pertenecen a la Comunidad Andina de Naciones (CAN), en donde ha existido una voluntad de impulsar y fortalecer la integración del mercado energético de la región. Ambos países intercambian energía eléctrica mediante transacciones spot de corto plazo, llamadas Transacciones Internacionales de Electricidad (TIE), buscando optimizar los recursos energéticos de los países, ya que la transacción ocurre cuando existen ventajas comparativas vía precio, es decir, el precio del país exportador es menor que el precio del país que demanda energía y también para respaldar déficits de energía eléctrica, cuando el país no tiene como cubrirlo con recursos propios. La iniciativa de la CAN en un comienzo pretendía la integración del mercado eléctrico; sin embargo, desde que comienzan las TIE en 2003 y hasta 2012, no han evolucionado en el aspecto integracionista como se preveía inicialmente (CAN, 2012; Glanchant y Pignon, 2003) y se concentraron en intercambios bilaterales de energía eléctrica entre ambos países.

Esto hace pertinente analizar cómo se forman los precios de las TIE, ya que, según las teorías que enmarcan la integración de mercados, cuando se tiende a este estado integrado, el resultado es que los precios convergen en el tiempo, lo que no ha ocurrido en el caso de los mercados eléctricos de Colombia y Ecuador. Por tanto, el objetivo de esta investigación es analizar la convergencia entre el precio de oferta de exportación de electricidad de Colombia (PONE) y el costo marginal de Ecuador (Precio en Bolsa). Esto se hace mediante un ejercicio econométrico utilizando análisis de componentes principales y el enfoque de beta convergencia. Se utilizan estas dos metodologías, dado que con la primera se mide el nivel de integración de los dos mercados analizados, mientras que con la segunda técnica se examina la disminución de la brecha de precios entre los dos mercados en el periodo analizado (Zachmann, 2008). Los principales resultados apuntan a la no convergencia de estos precios en los dos mercados analizados, lo cual también es corroborado de forma descriptiva a partir de las tendencias que muestran dichos precios. Como establece la CAN (2012), el intercambio de electricidad entre Colombia y Ecuador es una experiencia exitosa, pero no como un proceso de integración. ${ }^{1}$

La primera sección del artículo contiene una breve descripción de los mercados de energía eléctrica de Colombia y Ecuador, la cual tiende a ser comparativa, lo que ayuda a entender por qué por medio del tiempo Colombia se ha caracterizado por ser el país exportador. Luego se explica cómo ocurre la formación del

\footnotetext{
1 Ver también García (2006).
} 
precio de exportación de Colombia hacia Ecuador y se detalla cada uno de sus componentes. Finalmente se realizan dos ejercicios econométricos: Análisis de componentes principales y beta convergencia, los cuales pretenden demostrar que la brecha de precios de la energía eléctrica entre Colombia y Ecuador no se ha cerrado, sino por el contrario presenta tendencia creciente en el tiempo. En la última sección se concluye y se hacen algunas anotaciones finales acorde a los resultados encontrados.

\section{Características generales de los mercados eléctricos de Colombia y Ecuador}

Las TIE son transacciones horarias entre los mercados de corto plazo de los países interconectados, para este caso Colombia y Ecuador. Las TIE también se pueden definir mediante el modelo de acoplamiento o market splitting (Sierra \& Jaramillo, 2007), el cual consiste en un método de subasta implícita, segmentado en mercados cuya interconexión es limitada y obtiene precios marginales por áreas. Este precio determina la manera de remunerar por áreas. Bajo este modelo cada país conserva cierta autonomía en su regulación en cuanto a la formación de precios y las exportaciones e importaciones son determinadas vía precio. Estas transacciones tienen su origen en las diferencias de precios en los nodos frontera de los enlaces internacionales y son producto del despacho económico coordinado de los operadores de los sistemas de ambos países, en Colombia es XM-filial de ISA y en Ecuador el responsable es CENACE (Regulación CONELEC - 004/10). Según la Corporación Andina de Fomento (CAF, 2006) los beneficios que se obtienen de los intercambios de electricidad de los sistemas interconectados mejoran la competitividad en los mercados y la seguridad y la confiabilidad en el suministro del servicio. En teoría, entre las ventajas que traen las interconexiones de los sistemas eléctricos de los países se encuentran (CIER, 2006):

- Utilización óptima de los recursos energéticos, al tener en cuenta las complementariedades de las fuentes de generación entre ambos países, al igual que de la infraestructura eléctrica.

- Diversificación de la matriz energética de los países y el aprovechamiento de la complementariedad de recursos energéticos.

- Disminución de los precios de electricidad para el país importador y por ende de las tarifas cobradas a usuarios finales.

- Mejora en la seguridad, confiabilidad y calidad en el sistema de suministro de energía eléctrica. 
- Incentiva la competencia en la generación, dada la ampliación en la oferta; y una ampliación de la demanda del mercado, y por lo tanto la entrada de nuevos generadores, potencializando la inversión nacional y la inversión extranjera directa.

Las TIE constituyen el segundo escalón dentro de las posibilidades de integración de mercados eléctricos. La primera fase de integración consiste en transacciones por medio de contratos bilaterales entre países (caso Colombia Venezuela); la segunda corresponde al despacho coordinado, que para Colombia y Ecuador se denomina TIE; la tercera etapa implica el despacho integrado, es decir, unificación del despacho; la cuarta la constituye la integración regional, siendo indispensable para esta la presencia de un operador y administrador regional; y la etapa más avanzada estaría compuesta por la integración suprarregional, con una integración total de los mercados regionales.

Sin ir muy lejos geográficamente, se tiene como ejemplo el Sistema de Interconexión Eléctrica de los Países de América Central (SIEPAC), el cual se encuentra en la cuarta etapa de integración, con los mercados eléctricos de Guatemala, El Salvador, Honduras, Nicaragua, Costa Rica y Panamá. Allí existe un Mercado Eléctrico Regional (MER), el cual es un séptimo mercado con los seis sistemas nacionales existentes, con regulación regional por parte de la Comisión Regional de Interconexión Eléctrica (CRIE) y en donde se realizan transacciones internacionales de electricidad por medio del Ente Operador Regional (EOR) (EPR, 2012).

Como lo señala García \& Palacios (2006), el caso más avanzado en integración lo constituye el Nord Pool, el cual integra los mercados eléctricos de Noruega, Suecia, Finlandia y Dinamarca. Estos países de la región nórdica europea tienen un elevado consumo de energía eléctrica, dada su industria intensiva en energía y por las necesidades de calefacción que exige el clima de esa región. Dado lo anterior, la importancia de la seguridad del suministro energético incentivó a adelantar el proceso de integración de sus sistemas eléctricos, acompañado con las complementariedades en los recursos de generación que se presentan entre los mismos países. Las transacciones ocurren por medio de contratos bilaterales, acompañado con un mercado financiero creciente compuesto por futuros y opciones. En lo referente a los precios, estos han convergido a uno solo para el mercado como tal, en donde los países que inicialmente producían a altos costos (caso Finlandia y Suecia) se benefician de los que producen a menores costos (caso Noruega); los beneficios para el país con recursos de generación se resumen en la ampliación del mercado y el pago a un precio mayor de sus exportaciones.

En cuanto al marco histórico de las TIE entre Colombia y Ecuador, estas empiezan luego de que se firmara la Decisión CAN 536 en diciembre de 2002 
“Marco General para la Interconexión Subregional de Sistemas Eléctricos e Intercambio Intracomunitario de Electricidad", la cual constituye el marco jurídico que soporta la iniciativa integracionista de los mercados de energía eléctrica entre los países miembros. Desde 2003 se empiezan a dar efectivamente las transacciones de energía eléctrica entre Colombia y Ecuador, y hasta la fecha, bajo la modalidad y normativa de las TIE. Aunque de acuerdo con Sierra \& Jaramillo (2007), los intercambios de electricidad entre Colombia y Ecuador se han presentado desde antes del esquema TIE por medio de contratos bilaterales tal como se realizan transacciones con Venezuela en la actualidad. En noviembre de 2009 se aprueba la Decisión CAN 720, por la que se suspende la Decisión CAN 536 (excepto el Artículo 20) hasta por un período de dos años y durante dicho tiempo Colombia y Ecuador aplican el Régimen Transitorio, el cual consiste en la división de las rentas de congestión ${ }^{2}$ en 50 \% para cada país (se utilizan como instrumentos de redistribución, p.e. para electrificar zonas pobres que no tienen acceso al servicio), sin obligación alguna de vender energía en momentos de déficit del país exportador. Por último, la Decisión CAN 757 (2011) sustituye a la Decisión 720 e incorpora un régimen temporal para los intercambios eléctricos entre Ecuador y Perú, además del régimen bilateral transitorio para Colombia y Ecuador (CAN, 2012). En noviembre de 2011 los presidentes de los países miembros de la CAN manifestaron la voluntad de acelerar la integración eléctrica, por lo que es indispensable la interconexión física de los cuatro países miembros (Bolivia, Colombia, Ecuador y Perú) y la armonización regulatoria con miras a la conformación del bloque energético del Pacífico.

Como puede observarse en la figura 1, desde el comienzo de las TIE, Colombia se ha caracterizado por ser el país exportador; el nivel de importaciones es bastante reducido, llevándose a cabo solo en aquellos periodos que se hace necesario para satisfacer la demanda.

Dado que las TIE surgen del diferencial de precios en el nodo frontera, se evidencia que el costo de la energía eléctrica en Colombia es menor que el de Ecuador, lo que puede explicarse en los recursos de generación que utiliza cada uno de los países. En el caso de Colombia, se encuentra que para 2011 el 77\% de la oferta de energía eléctrica fue hidráulica y $16 \%$ térmica, lo que se muestra en la figura 2.

2 Rentas de Congestión: Rentas económicas originadas por la diferencia entre el precio de oferta del mercado exportador y el precio de mercado de corto plazo delimportador en los extremos de cada enlace internacional. Para el caso del Ecuador, el precio de mercado de corto plazo corresponde al costo de mercado de corto plazo (REGULACIÓN No. CONELEC - 004/10). 


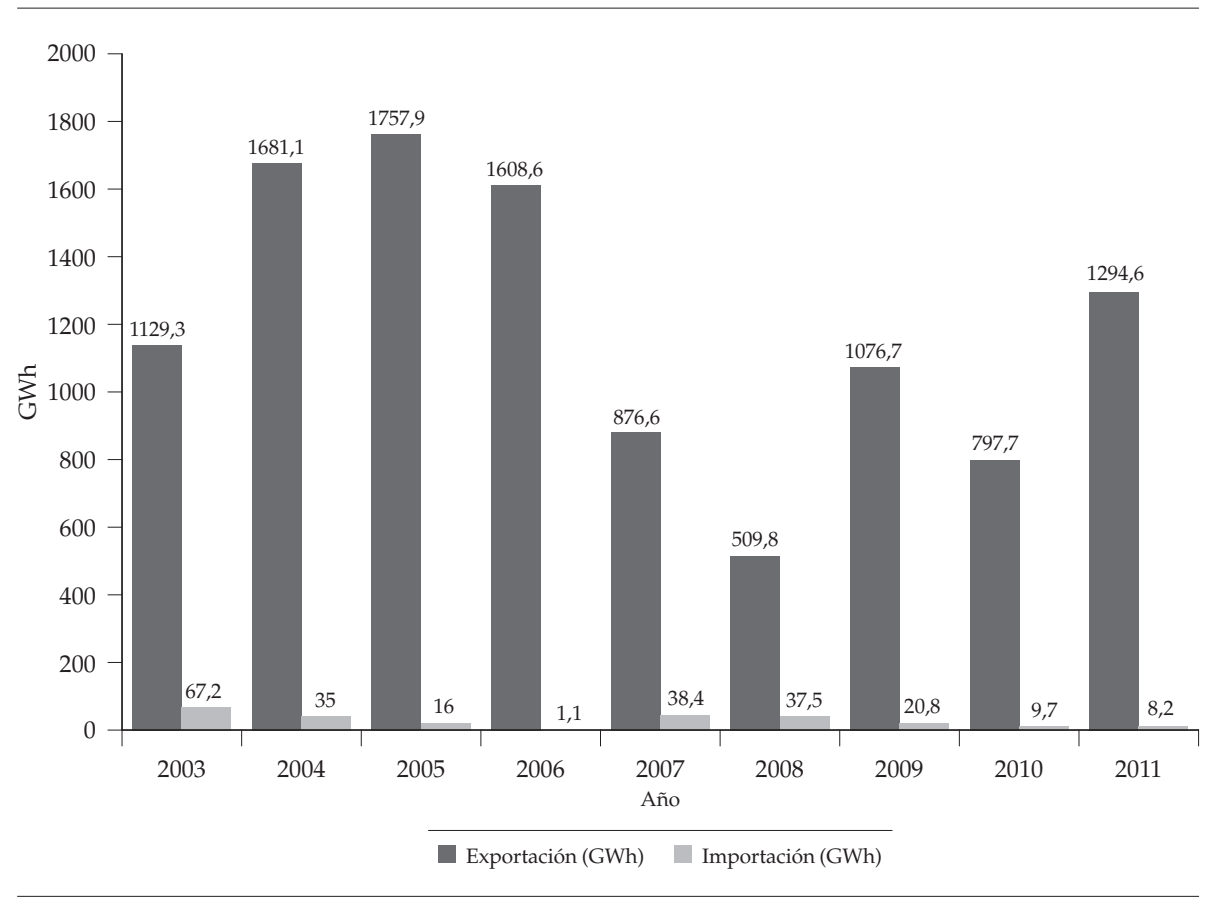

Figura 1. Transacciones internacionales de electricidad Colombia - Ecuador (2003-2011)

Fuente: XM (2012a).

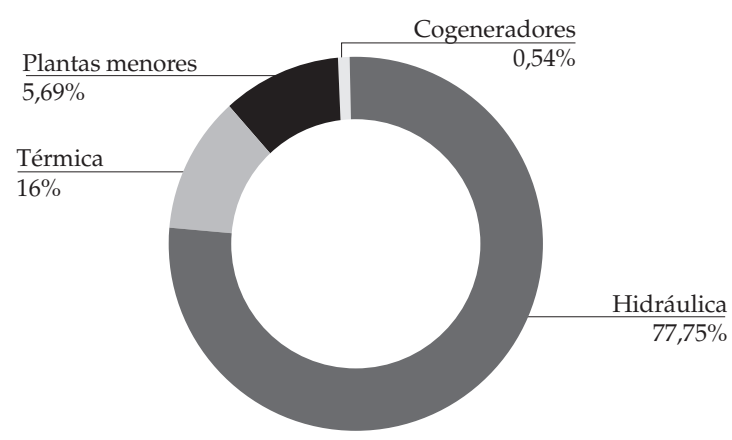

Figura 2. Producción de energía eléctrica en Colombia, 2011

Fuente: XM (2012a).

En cuanto a la producción de energía eléctrica en Ecuador, se encuentra que para 2011 se comportó de la siguiente manera (figura 3): cerca de un $59 \%$ fue con recursos hídricos, alrededor del $7 \%$ correspondió las importaciones desde Colombia y el resto corresponde a generación térmica con una alta composición de 
combustibles dependientes del precio del petróleo, participando el gas (recurso más económico que los anteriores) con un porcentaje cercano al $4 \%$. Sin embargo, es importante recalcar que, según CENACE (2012), la producción térmica disminuyó en 2011 un 23,26 \% respecto de 2010 y argumenta que la producción hidráulica que ocurrió durante 2011 es mayor que la media histórica, siendo en 201130 \% mayor respecto de 2010. Las importaciones de Ecuador provenientes de Colombia aumentaron entre 2010 y 2011 en un 62,3\%.

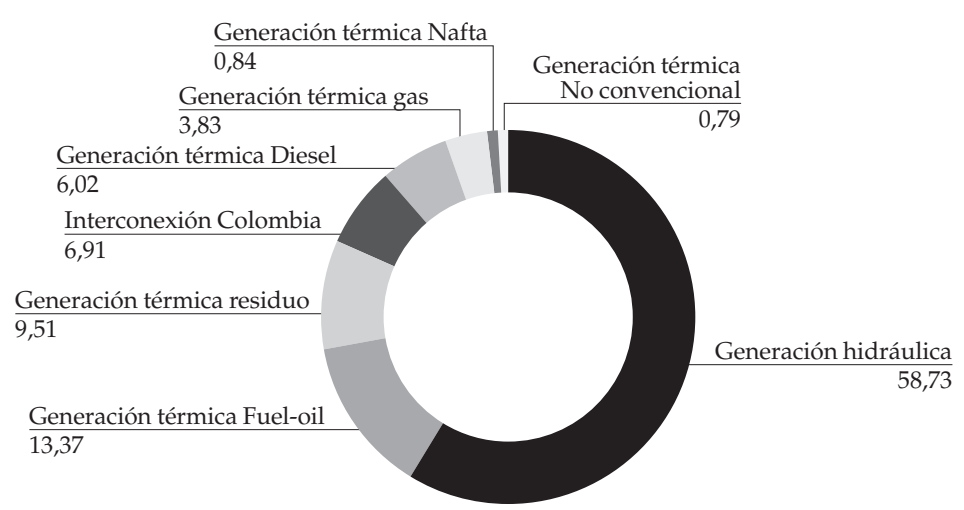

Figura 3. Producción de energía eléctrica en Ecuador, 2011

Fuente: CENACE (2011).

Los costos de generación de energía eléctrica son consecuencia de la tecnología que se utiliza para producirla, siendo los recursos hídricos más económicos comparativamente con los térmicos; es más, aún dependen del precio del petróleo, siendo el gas la opción de menor costo en este grupo de generadores térmicos. Dado esto, en Ecuador hay una vulnerabilidad, ya que la generación con recursos térmicos ha sido históricamente importante y esta ocurre principalmente con combustibles líquidos, tal como se evidencia en la figura 4.

En Colombia la generación con recursos térmicos se ha caracterizado por la alta utilización de gas natural, seguida por el carbón, tecnologías más económicas que los combustibles líquidos utilizados en Ecuador, tal como puede verse en la figura 5.

Dado lo anterior, Ecuador ha sido importador neto de energía eléctrica desde el comienzo de las TIE hasta 2012, tanto por la seguridad en el suministro como por la diferencia en tecnología que utiliza para la generación de energía eléctrica, como se evidencia en la breve descripción de los mercados de la tabla 1. 


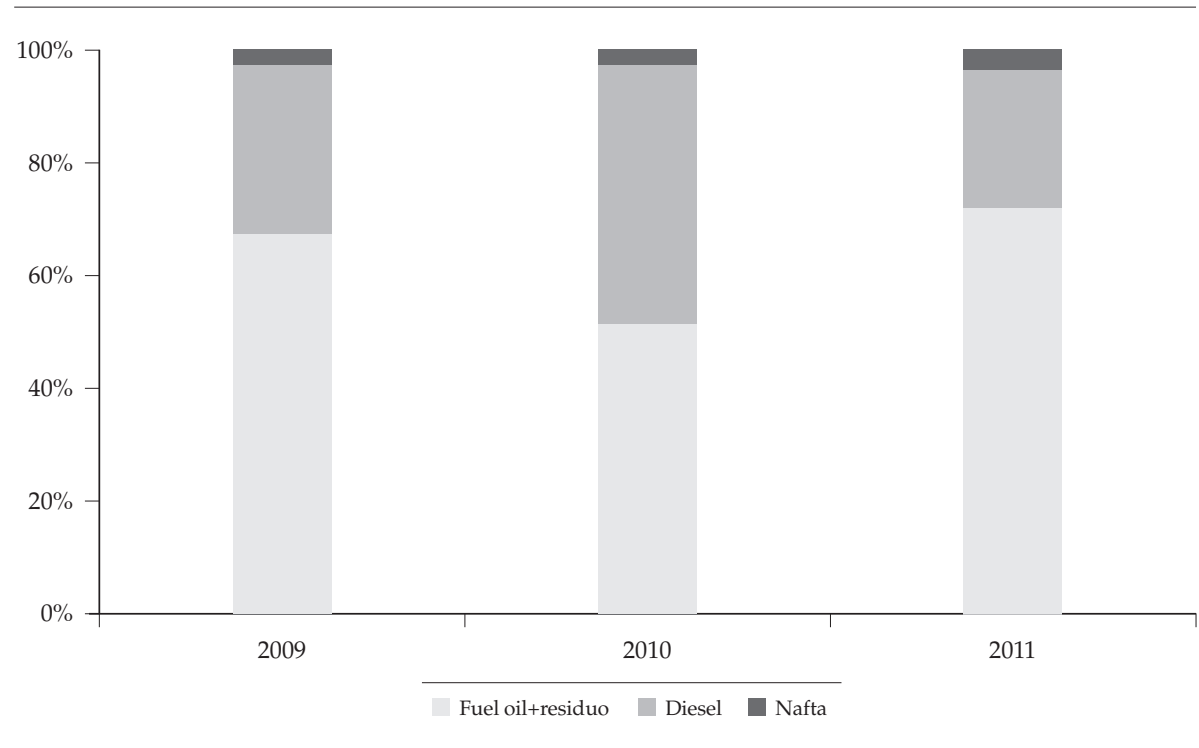

Figura 4. Consumo de combustibles para la generación de energía eléctrica en Ecuador Fuente: CENACE $(2012,2011,2010)$.

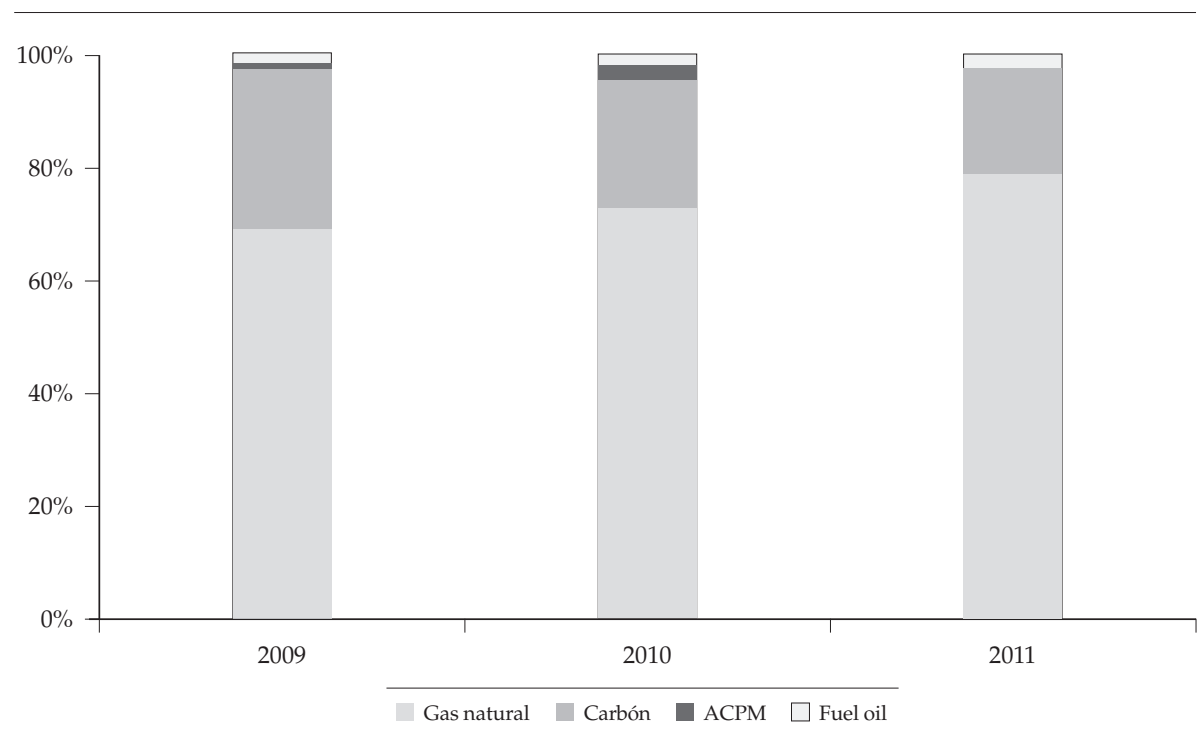

Figura 5. Consumo de combustibles para la generación de energía eléctrica en Colombia

Fuente: XM (2012a). 
Tabla 1. Breve caracterización de los mercados de energía eléctrica Ecuador - Colombia, 2011

\begin{tabular}{lrr}
\hline \multicolumn{1}{c}{ Variable } & \multicolumn{1}{c}{ Ecuador } & Colombia \\
\hline Demanda interna (GWh) & 17747,80 & 57150 \\
Producción bruta total (GWh) & 18732,33 & 58620,4 \\
Producción hidráulica (GWh) & 11002,20 & 45583,1 \\
& $(58,73 \%)$ & $(77,75 \%)$ \\
Producción térmica (GWh) & 6288,27 & 9383,7 \\
& $(33,57 \%)$ & $(16 \%)$ \\
Producción otros & 147,27 & 3653,6 \\
Exportación de electricidad (GWh) & $(0,78 \%)$ & $(6,23 \%)$ \\
Importación de electricidad (GWh) & 8,22 & 1294,59 \\
Cobertura & 1294,59 & 8,22 \\
\hline
\end{tabular}

Fuente: Elaboración propia con datos de CENACE; XM (2012a).

En el mercado de energía eléctrica colombiano se debe garantizar primero que todo el cubrimiento de la demanda nacional, usando los recursos con menor precio de oferta, seguido de la demanda TIE Ecuador, usando el exceso de capacidad de algún recurso ya despachado o, en caso de ser necesario, utilizando un recurso más caro, y por último las exportaciones a Venezuela que se despachan con los recursos siguientes, como se puede observar en la figura 6, lo descrito anteriormente es lo que se denomina el despacho ideal o despacho por orden de mérito.

Los generadores que entraron en el despacho ideal y abastecieron la demanda nacional se remuneran con base en el MPO (Máximo Precio Ofertado) nacional, el cual es el precio de oferta del recurso más costoso que fue necesario para atender dicha demanda, este se diferencia del precio de bolsa en que tiene en cuenta un diferencial que corresponde a la remuneración de los arranques de los recursos térmicos. Los generadores que atendieron demanda TIE se remuneran al MPO-TIE, el cual es el precio de oferta del recurso más costoso que fue necesario para atender la demanda nacional más TIEs; para Venezuela se tiene el MPO internacional. Por otro lado, está la reconciliación fuera de mérito que son generadores de reserva que estuvieron prendidos, pero no despachados (que corresponden al recurso 8 en la figura 6), y por último una importación de Colombia se considera una generación por lo que entra en el despacho ideal. 


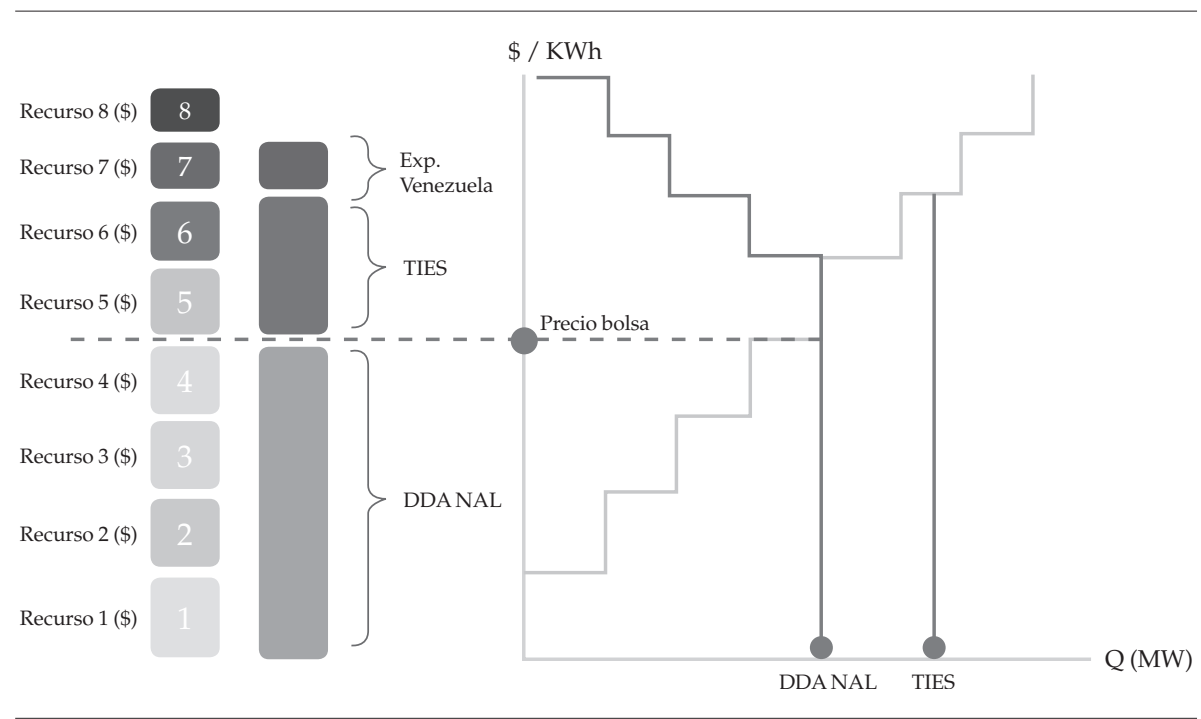

Figura 6. Despacho energía eléctrica en el mercado eléctrico colombiano Fuente: Elaboración propia.

Es importante resaltar que no solo la necesidad de garantizar el cubrimiento de la demanda de energía eléctrica en un país, o el hecho de que un país vecino genere energía eléctrica a un costo menor dada la tecnología disponible, son las condiciones suficientes para que ocurra la transacción internacional de electricidad. Al respecto, hay otros dos factores claves: por un lado está la interconexión física (redes) y por el otro la armonización regulatoria, que se evidencia en las leyes del sector eléctrico y con las Resoluciones de los reguladores.

Con respecto a las redes, Colombia y Ecuador cuentan con tres interconexiones, las cuales se muestran en la tabla 2, y la figura 7 ilustra las interconexiones de Colombia con sus países vecinos, siendo este país estratégico por su posición geográfica la puerta para interconectar América del Sur con SIEPAC.

Tabla 2. Interconexiones eléctricas Colombia - Ecuador

\begin{tabular}{lccc}
\hline & Fecha de entrada & KV & MW \\
\hline Panamericana - Tulcán & 1998 & 138 & 35 \\
Jamondino - Pomasqui 1 y 2 & 2003 & 230 & 250 \\
Jamondino - Pomasqui 1, 2, 3 y 4 & 2007 & 230 & 500 \\
Capacidad total Colombia - Ecuador & & & $585 \mathrm{MW}$ \\
\hline
\end{tabular}

Fuente: Camargo (2011). 


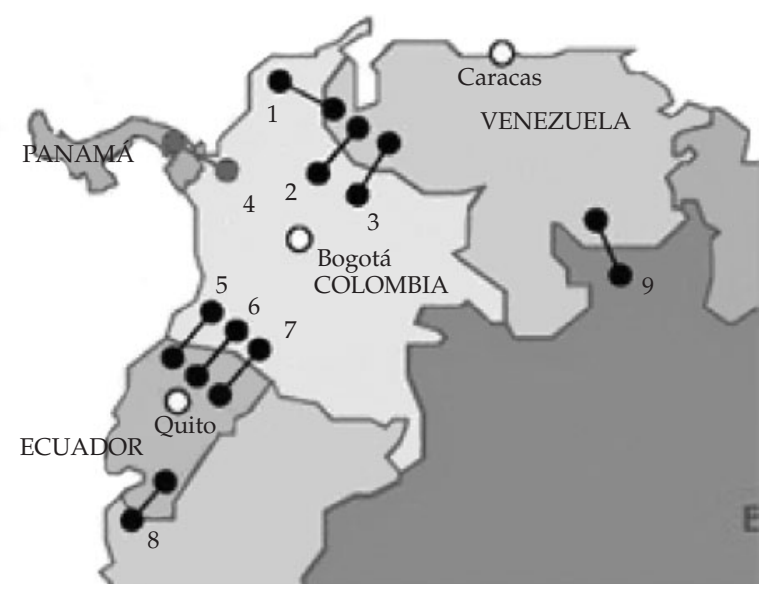

Figura 7. Interconexiones Colombia con los países vecinos Fuente: CIER (2011).

Otro aspecto fundamental en las TIE lo constituye el marco legal de los mercados de electricidad en cada uno de los países. Se requiere una armonización regulatoria como premisa indispensable para que se hagan efectivas dichas transacciones bilaterales entre países. La tabla 3 resume las principales leyes y resoluciones para Colombia, en donde la Resolución 004 de 2003 establece la regulación aplicable a las TIE y que es complementada por la Resolución 014 de 2004.

Tabla 3. Marco normativo Colombia

Ley 142, 1994. Art. 23

Ley 143,1994 . Art. 34

RESOLUCIÓN CREG 004 - 2003

CREG 006 - 2003

RESOLUCIÓN 014 - 2004

RESOLUCIÓN 160 - 2009
Fija la política en cuanto al intercambio internacional de electricidad.

Asigna funciones al Centro Nacional de Despacho (CND), entre las que se encuentra ejercer la coordinación, supervisión, control y análisis de la operación de los recursos de generación, interconexión y transmisión incluyendo las interconexiones internacionales.

Regulación TIE Corto plazo.

Normas sobre registro de fronteras comerciales.

Planeación, coordinación, supervisión y control operativo de los enlaces internacionales.

Por la cual se adopta la regulación aplicable a las Transacciones Internacionales de Electricidad de Corto Plazo TIE, entre Colombia y Ecuador, de conformidad con el Régimen Transitorio adoptado por la Decisión CAN 720.

Fuente: Elaboración propia, con datos CREG. 
Para el caso de Ecuador, la tabla 4 muestra un resumen de las leyes más importantes y que involucran las TIE, la Regulación 004/10 se encarga del desarrollo de las TIE en el periodo de vigencia de la Decisión CAN 720.

Cada país por separado tiene su propia legislación, sin embargo, al estar Colombia y Ecuador inmersos en la Comunidad Andina de Naciones (CAN), ambos están sujetos a disposiciones supranacionales, tales como el Acuerdo de Cartagena, que dio su origen en 1996; la Decisión CAN 536 de 2002, la Decisión CAN 720 de 2009 y la vigente transitoriamente Decisión CAN 757. Estas se resumen en la tabla 5 .

En la actualidad se encuentra este marco jurídico bajo construcción y se opera con condiciones transitorias, ya que se está construyendo un marco general que tiene en cuenta el marco regulatorio andino existente y la legislación de cada país miembro de la comunidad.

Tabla 4. Marco normativo Ecuador

Ley del Régimen del Sector Eléctrico, 1996

Aplicación del Mandato Constituyente No. 15 Regulación No. CONELEC 006/08

CONELEC - 004/10
Contiene las normas relacionadas con la estructura del sector eléctrico y de su funcionamiento.

Establecer los parámetros regulatorios específicos para el establecimiento de una tarifa única que deben aplicar las empresas eléctricas de distribución para cada tipo de consumo de energía eléctrica. Relacionado: Generación, Distribución, Mercado Eléctrico Mayorista, Transmisión, Grandes Consumidores, Transacciones Internacionales y Tarifas.

Desarrollo de las Transacciones Internacionales de Electricidad en el periodo de vigencia de la Decisión 720 de la Comunidad Andina.

Fuente: Elaboración propia con datos CONELEC.

Tabla 5. Marco normativo TIES - CAN

Acuerdo de Cartagena (1996)

Decisión CAN 536 (2002)

Decisión CAN 557 (2003)

Decisión CAN 720 (2009)

Decisión CAN 757 (2011)
Acuerdo que tiene por objetivo promover el desarrollo equilibrado y armónico de los países miembros en condiciones de equidad, mediante la integración y la cooperación económica y social.

Marco jurídico comunitario para impulsar el desarrollo del tema eléctrico en los países miembros.

Creación del Consejo de Ministros de Energía, Electricidad, Hidrocarburos y Minas de la Comunidad Andina.

Se suspende por dos años la Decisión CAN 536, para establecer un nuevo marco general para los intercambios de energía eléctrica entre los países miembros.

Sustituye la Decisión 720 e incorpora un régimen temporal para los intercambios eléctricos entre Ecuador y Perú, además del régimen bilateral transitorio para Colombia y Ecuador. 


\section{Formación del precio TIE}

Existiendo la interconexión eléctrica, el marco regulatorio que posibilita las TIE y entendiendo por qué Colombia, desde 2003 hasta la fecha, se ha caracterizado por ser el país exportador, se procede a analizar el tema de la operación de las TIE, prestando especial interés en la formación del PONE (Precio de Oferta en Nodo Exportador). La manera de operación de las TIE se basa en la comparación de precios en los nodos frontera. De esta manera, para que Ecuador importe energía (caso predominante por medio del tiempo) debe cumplirse la desigualdad de la ecuación 1 :

$$
\text { POI }-P_{\text {umbral }}>\text { PON E }
$$

Fuente: CONELEC 004/10.

En donde POI corresponde al Precio de Oferta de Importación de Ecuador, ${ }^{3}$ PONE es el precio de oferta de exportación de Colombia y $P_{\text {umbral }}$ es el valor que se determina como un porcentaje del precio de oferta formado en el nodo frontera del país importador y que sirve para dar un margen de seguridad a la decisión del sentido de la transacción a efectuarse. El PONE Colombia se calcula por medio de la ecuación:

$$
P O N E C o l=P_{b}+C M R+C R A E+C P+C U+\text { Serv }
$$

Y debe garantizar que se cubran los costos de Colombia como puede observarse en la figura 8.

Dado que el PONE es quien finalmente da la dirección de la transacción (exportación o importación) es pertinente explicar cada uno de sus componentes:

- El precio de bolsa $\left(P_{b}\right)$ : se calcula con base en los precios de oferta enviados por los generadores, caso contrario a América Latina donde en general se envían son los costos de generación, este precio de oferta depende de la disponibilidad de los recursos en especial de las unidades más grandes de generación hidráulica.

3 Incluye el costo marginal del mercado ecuatoriano, que es función de los costos de generación, los costos de transmisión, costos asociados de la remuneración de arranque de las plantas de turbo-vapor más otros costos. 


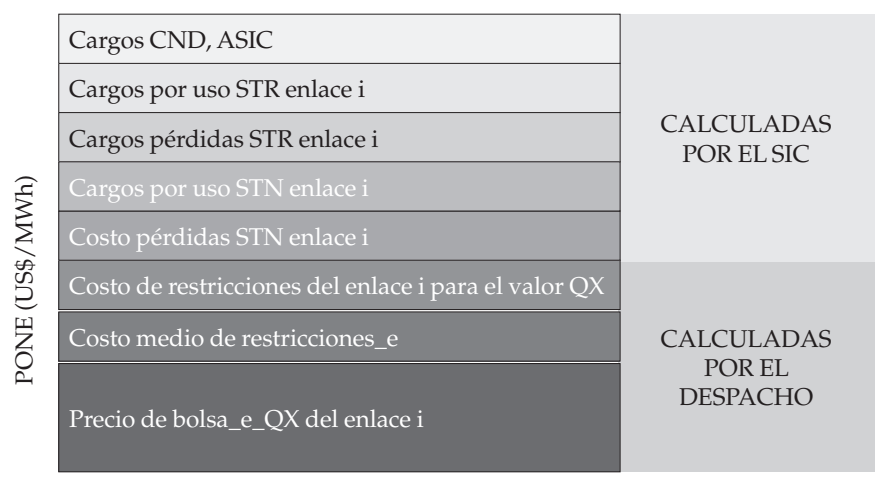

Figura 8. Componentes PONE Colombia

Fuente: XM (2012b).

- El costo medio de las restricciones (CMR): está dado por las reconciliaciones que suceden en el mercado eléctrico por motivo de la diferencia entre la operación real y la operación ideal; esta última se realiza con base en los precios ofertados y unas cantidades dadas y al compararlo con la demanda programada se obtiene el precio de bolsa. Por su parte, la operación real considera todos los ajustes pertinentes para satisfacer la demanda en tiempo real. Dado lo anterior, si la operación real es mayor a la ideal se genera una reconciliación positiva, es decir, se le paga al generador el valor de dicha diferencia.

- El costo de restricción asociada al enlace (CRAE): son las restricciones propias de la exportación a Ecuador.

- Los costos de pérdidas del Sistema de Transmisión Nacional (STN) ${ }^{4}$ y Sistema de Transmisión Regional (STR $)^{5}(\mathrm{CP})$ : corresponden a la diferencia entre la generación total y la demanda total por condiciones técnicas, como la pérdida por calor, y las pérdidas no técnicas, que se refieren a las conexiones ilegales al sistema.

- El cargo por uso del STN y STR (CU): es el costo de la línea de transmisión que se cobra no con base en la magnitud de uso de esta, sino dada una remuneración a la inversión realizada.

- Los servicios del Centro Nacional de Despacho (CND) y Administrador del sistema de intercambios comerciales (ASIC) (Serv): son los cargos por administración y operación del mercado que para el caso de Colombia los realiza la compañía XM.

4 Líneas de transmisión de tensión mayor a 220 voltios.

5 Líneas de transmisión de tensión menores a 220 voltios. 
El PONE, a su vez depende de la cantidad de energía eléctrica que requiere Ecuador. Dado esto Colombia genera una curva de oferta, la cual es monotónicamente creciente, es decir, a mayor cantidad entran recursos más costosos lo que aumenta el valor del PONE, hasta el punto en donde la cantidad es exactamente igual a la capacidad total del enlace (500 MW), que en la figura 9 corresponde al valor de $Q_{t}$.

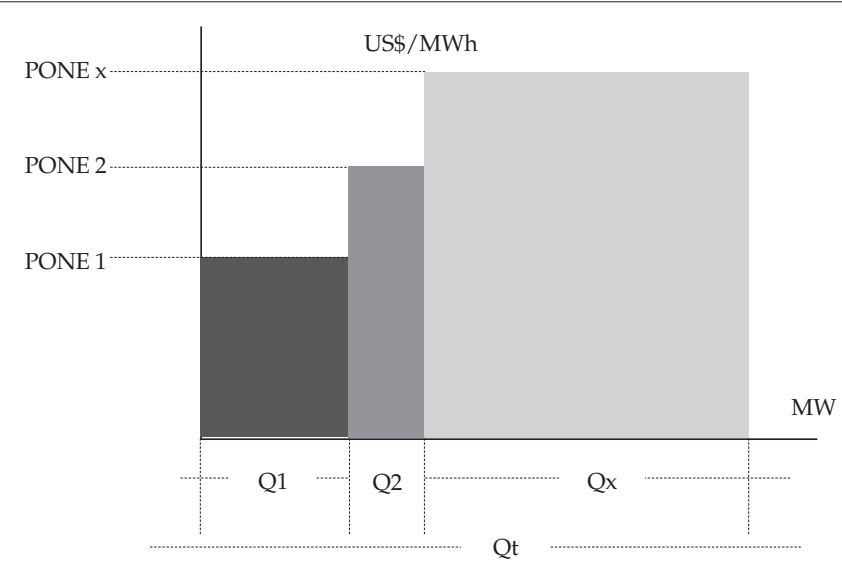

Figura 9. Escalones de precio de oferta PONE

Fuente: XM (2012b).

Luego de realizarse la comparación de precios, el sistema o país que importa realiza el despacho económico programado, que se define como el programa diario de los recursos de generación del sistema interconectado nacional para atender la demanda con calidad, confiabilidad, seguridad y economía (XM, 2012b), con la oferta disponible en el enlace internacional, determinan y comunican las cantidades a importar al país exportador, para que este último haga la programación teniendo en cuenta estas cantidades (CONELEC, 2010).

La liquidación de la TIE se realiza con la lectura del medidor en el nodo exportador, dadas las pérdidas que pueden presentarse. La factura se hace un mes después $(m+1)$, el día 10 , en donde ya se ha realizado el intercambio de información de precio y cantidades, ver figura 10. Así, la manera de operar las TIE cronológicamente comienza un día antes con el despacho económico coordinado, se hace el despacho real el día de la operación y luego se generan en el mismo mes dos versiones de la liquidación (TX1 y TX2), el mes siguiente a la operación se envía un primer resumen de la facturación (TXRm) y finalmente el día 10 de este mes se realiza la facturación final, donde Ecuador paga a Colombia el máximo precio entre el PONE Colombia y el Costo Marginal de Ecuador, dada la lectura del nodo y se cancela en dólares a la TRM del día (Zapata, et al., 2012). 


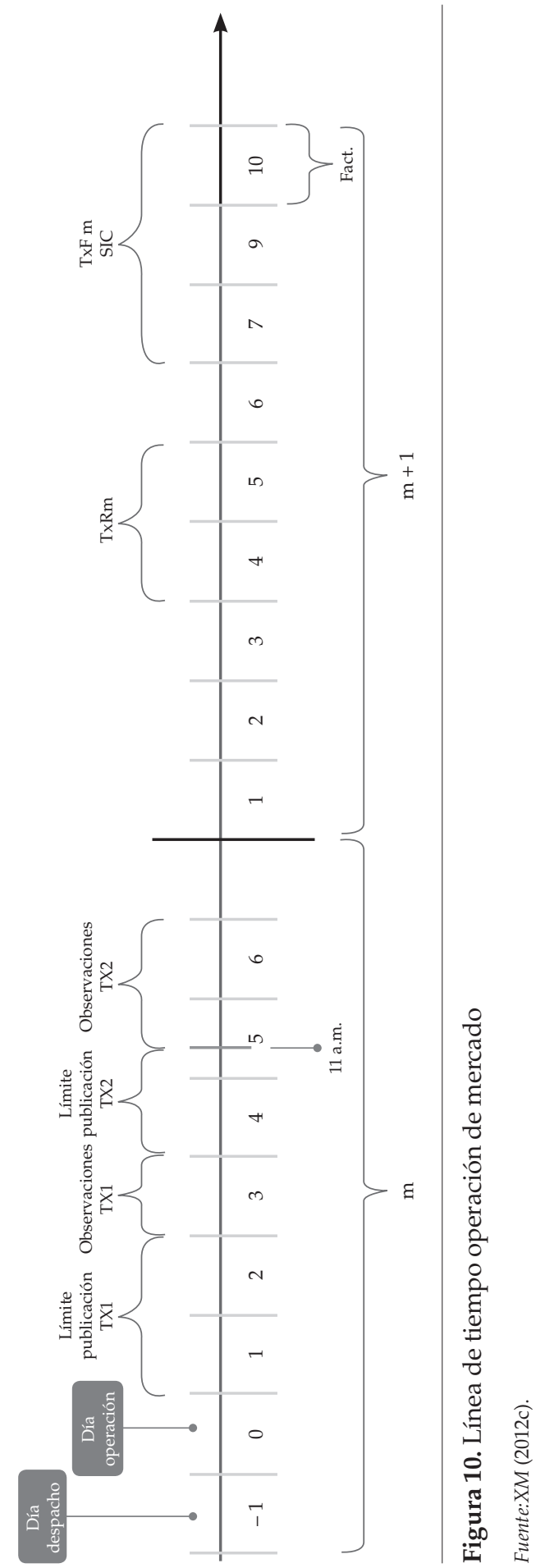




\section{Convergencia en precios de las TIE}

Dado que en principio el objetivo principal de la decisión CAN 532 de 2002 era lograr la integración entre Colombia y Ecuador, se analiza si este se ha logrado, al hacer un análisis de convergencia entre los precios PONE y el costo marginal de Ecuador; se dejan por fuera el precio POI y el precio de bolsa colombiano por la baja cantidad de importaciones de energía de parte de Colombia desde Ecuador. Este análisis se hace entre el precio PONE y el Costo marginal, debido a que se asume que por la integración entre los países, esto se debe ver reflejado en los precios que perciben los consumidores del país con costos más altos, el cual está relacionado con el costo marginal, mientras que el POI no afecta a los consumidores ecuatorianos. Este análisis se hace con datos de precios y cantidades de exportación e importación proporcionados por la empresa XM.

En primer lugar se hace una breve estadística descriptiva de lo encontrado; en la figura 11 se muestran las diferencias entre los nodos y el costo marginal del mercado ecuatoriano y se puede ver que este no se está acercando a cero por medio del tiempo ni para ECCU $3^{6}$ (figura izquierda), ni para ECCU47 (figura derecha), además se hace la correlación entre los precios de los nodos y el precio de bolsa de Ecuador (ver tabla 6) y se separa por tipo de demanda (ver tabla 7), estos muestran que realmente no ha existido una correlación entre los precios PONE (ECCU 3 y ECCU 4) y el precio del mercado ecuatoriano.
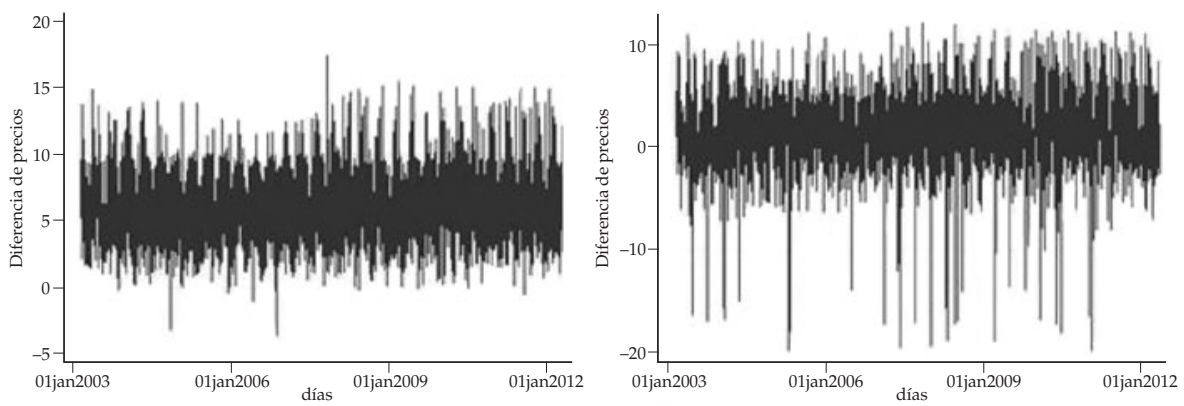

Figura 11. Convergencia PONE

Fuente: Elaboración propia con datos proporcionados por XM.

6 Enlace de Colombia Ecuador de $138 \mathrm{kV}$.

7 Enlace de Colombia Ecuador de $230 \mathrm{kV}$. 
Tabla 6. Correlación

\begin{tabular}{cccc}
\hline & ECCU 3 & ECCU 4 & Cmg Ecu \\
\hline ECCU 3 & 1 & & \\
ECCU 4 & 0,1489 & 1 & \\
Cmg Ecu & 0,1441 & 0,0869 & 1 \\
\hline
\end{tabular}

Fuente: Elaboración propia.

Tabla 7. Correlaciones por tipo de demanda

\begin{tabular}{cccc}
\hline \multicolumn{5}{c}{ Demanda Alta } & Cmg Ecu \\
\hline ECCU 3 & 1 & ECCU 4 & \\
ECCU 4 & $-0,0804$ & 1 & 1 \\
Cmg Ecu & 0,1062 & 0,112 & \\
\hline \multicolumn{5}{c}{ Demanda Media } \\
\hline ECCU 3 & ECCU 3 & ECCU 4 Ecu \\
ECCU 4 & 1 & 1 & 1 \\
Cmg Ecu & $-0,1574$ & 0,2261 & \\
\hline & 0,093 & & Cmg Ecu \\
\hline ECCU 3 & \multicolumn{2}{c}{ Demanda Baja } & \\
\hline ECCU 4 & ECCU 3 & 1 & 1 \\
Cmg Ecu & $-0,0651$ & 0,2699 & \\
\hline
\end{tabular}

Fuente: Elaboración propia.

Para corroborar esto, se hace un análisis de componentes principales como el propuesto por Evans, et al. (2008), este análisis busca explicar la mayor cantidad de la variación con el menor número de componentes posibles, haciendo un análisis de los valores propios de la matriz de correlaciones, que como vimos en el caso anterior era muy baja; los componentes se organizan del que más explica al que menos explica esperando que con pocos componentes se explique mucha variación, mientras más componentes se necesiten se interpreta que el mercado tiene más segmentos. Las variables aquí utilizadas tienen en cuenta, al igual que Evans, et al. (2008), los efectos estacionales a los que están sujetos los precios de la energía, especialmente los colombianos que dependen altamente de los veranos e inviernos que suceden en el país debido a su capacidad hídrica, 
los resultados se presentan a continuación tanto para el caso conjunto (tabla 8) como por tipo de demanda (tabla 9):

Tabla 8. Componentes principales

\begin{tabular}{ccccc}
\hline Component & Eigenvalue & Difference & Proportion & Cumulative \\
\hline Comp1 & 1,25515 & 0,341979 & 0,4184 & 0,4184 \\
Comp2 & 0,913172 & 0,0814969 & 0,3044 & 0,7228 \\
Comp3 & 0,831676 & & 0,2772 & 1 \\
\hline
\end{tabular}

Fuente: Elaboración propia.

Tabla 9. Componentes principales por tipo de demanda

\begin{tabular}{ccccc}
\hline \multicolumn{5}{c}{ Demanda Alta } \\
\hline Component & Eigenvalue & Difference & Proportion & Cumulative \\
\hline Comp1 & 1,11948 & 0,0393409 & 0,3732 & 0,3732 \\
\hline Comp2 & 1,08014 & 0,27976 & 0,36 & 0,7332 \\
\hline Comp3 & 0,800379 & $\cdot$ & 0,2668 & 1 \\
\hline Component & Eigenvalue & Difference & Proportion & Cumulative \\
\hline Comp1 & 1,23824 & 0,152467 & 0,4127 & 0,4127 \\
\hline Comp2 & 1,08577 & 0,40979 & 0,3619 & 0,7747 \\
\hline Comp3 & 0,675984 & $\cdot$ & 0,2253 & 1 \\
\hline Component & Eigenvalue & Difference & Proportion & Cumulative \\
\hline Comp1 & 1,29483 & 0,235814 & 0,4316 & 0,4316 \\
\hline Comp2 & 1,05901 & 0,41285 & 0,353 & 0,7846 \\
\hline Comp3 & 0,646162 & Demanda Maja & 0,2154 & 1 \\
\hline
\end{tabular}

Fuente: Elaboración propia.

Esto corrobora lo encontrado con las correlaciones, ya que el primer componente solo explica el 41 \% de la variación y con el segundo solo llega a explicar un $72 \%$ de esta, esto implica que la reducción de dimensionalidad buscada por este método, es decir, el mercado está segmentado en tres partes, lo que implica una no convergencia de los precios, y, aun separándolo por tipos de demanda el mercado, sigue estando segmentado en tres partes, sin importar si fuese baja, media o alta demanda. 
Por último, se hace un análisis de beta convergencia el cual busca mostrar que por medio del tiempo las diferencias entre los precios han ido disminuyendo, si bien el análisis de beta convergencia se ha utilizado en la literatura sobre crecimiento económico para examinar si los países menos desarrollados están haciendo catching up con respecto a los países industrializados, ver Barro, \& Sala-i Martin (1992), Zachmann (2008) y Wolszczak-Derlacz (2008), quienes han mostrado que esta metodología también puede ser utilizada para hacer un análisis de disminución de brechas dado que los costos de transporte, en este caso, están siendo incluidos vía restricciones. Esto se hace calculando las diferencias entre los precios y explicarlo con respecto a su rezago (para hacer esto la variable en diferencias debe de ser estacionaria, lo cual se cumple en el caso de los precios), en caso tal que el beta asociado con la variable sea significativo y negativo, esto implica convergencia en precios entre los nodos y el costo marginal ecuatoriano (Wolszczak-Derlacz, 2008), los resultados de esta estimación se presentan en la tabla 10 (para ver la beta convergencia separada por tipo de demanda referirse la tabla 11), lo que muestra este análisis es congruente con lo anterior, es decir, no existe convergencia en precios entre el PONE y el precio del mercado ecuatoriano, es más las diferencias han ido creciendo por medio del tiempo dado que el beta del rezago da positivo y significativo (esto se puede ver en la figura 11) esto puede deberse al hecho de que por regulación al haber exportaciones, que son frecuentes, se paga el máximo entre el precio del mercado y el precio del PONE por lo cual existen incentivos para poner el precio más alto ya que igualmente se está despachando a ese precio y al igual que el análisis de componentes principales esto se mantiene para todo tipo de demanda.

Tabla 10. Beta convergencia

\begin{tabular}{ccc}
\hline & Diff ECCU 3 & Diff ECCU 4 \\
\hline \multirow{2}{*}{ Rezago } & 0,121 & 0,001 \\
& $(0,017)$ & $(0,017)$ \\
Constante & 0,377 & $-5,213$ \\
& $(0,055)$ & $(1,019)$ \\
\hline
\end{tabular}

Fuente: Elaboración Propia. 
Tabla 11. Convergencia por nodo

\begin{tabular}{cccc}
\hline \multirow{4}{c}{ Nodo ECCU3. Diferencia } & \\
\hline \multirow{2}{*}{ Rezago } & Dda Alta & Dda Media & Dda Baja \\
\cline { 2 - 4 } & 0,691 & 0,751 & 0,763 \\
\multirow{2}{*}{ Constante } & $(0,012)$ & $(0,011)$ & $(0,0111)$ \\
& 2,12042 & 1,539456 & 1,14645 \\
& $(0,092)$ & $(0,078)$ & $(0,067)$ \\
\hline \multirow{2}{*}{ Rezago } & Nodo ECCU 4. Diferencia & Dda Baja \\
\hline \multirow{2}{*}{ Constante } & 0,144 & Dda Media & 0,491 \\
& $(0,017)$ & 0,833 & $(0,015)$ \\
& 0,680 & $(0,009)$ & 0,426 \\
& $(0,209)$ & 0,141 & $(0,092)$ \\
\hline
\end{tabular}

*Los valores en paréntesis son el Error Std.

Fuente: Elaboración propia.

No obstante, otras alternativas metodológicas, como análisis de cointegración y filtro de Kalman pueden ser utilizadas para examinar si los dos países comparten las fluctuaciones en precios, disminuyendo la brecha entre ambos mercados. Sin embargo, los métodos utilizados en este trabajo son coherentes con el objetivo de esta investigación y se dejan como posibles trabajos futuros la aplicación de estas otras metodologías.

\section{Conclusiones}

Una de las principales ventajas que presenta el mercado eléctrico colombiano, comparativamente con el ecuatoriano, es que como en Colombia la principal fuente de generación son los recursos hídricos, dada la riqueza de agua que posee, los costos de generación en este país son menores, haciendo a su vez que el precio de generación sea menor. Ecuador, por su parte, a pesar de que parte de su componente tecnológico es hídrico, la participación de generación térmica es importante, para lo cual utiliza combustibles líquidos dependientes del precio del petróleo con el fuel oil y el diesel, y por lo tanto, los costos de generación son mayores. Dado esto, Colombia ha sido un exportador neto de electricidad a Ecuador. 
Los intercambios de energía eléctrica entre Colombia y Ecuador empiezan en 2003, luego de la firma de Decisión CAN 536 en 2002, la cual buscaba impulsar el proceso de integración en la región, incluyendo el sector eléctrico. No obstante, estos dos países se han concentrado en las transacciones internacionales de electricidad (García, 2006). Una de las bondades de la integración y de estas transacciones de electricidad, además de la garantía de suministro, se sustenta en la convergencia de precios haciendo que aquellos mercados con ventajas comparativas en costos exporten el servicio a aquellos mercados con mayores costos, lo que hace que el precio en ambos mercados tienda a ser muy similar. Por supuesto, esto requiere de la interconexión física y la armonización regulatoria.

En el caso de las TIE entre Colombia y Ecuador, a partir de los ejercicios empíricos, realizados por medio de un análisis de componentes principales y un enfoque de beta convergencia los precios no han convergido en sus diez años de funcionamiento, por el contrario, el ejercicio muestra que los precios en cada país cada vez se distancian más.

Colombia opera por medio del despacho económico coordinado, y en este esquema debe satisfacer inicialmente las necesidades internas con los recursos más económicos y luego proceder a suministrar energía eléctrica a Ecuador, utilizando lógicamente recursos más costosos, ya que el despacho se realiza por orden de mérito. Se percibe que en la medida de que ambos mercados sean separados, no integrados (un solo mercado), a pesar de que el país con la tecnología más costosa perciba beneficios vía la prestación del servicio nacional por medio de importaciones con un recurso más económico; sin embargo, es muy difícil hablar de una convergencia en precios sin desconocer el efecto positivo en términos de confiabilidad de suministro de este.

Para hablar de integración de mercados eléctricos, por ejemplo como lo ha hecho el Nord Pool se requiere avanzar no solo desde el punto de vista institucional y normativo, sino que es necesario la conformación de entes supranacionales como un único ente operador del sistema y regulador, así se apoyen en los entes existentes en cada mercado. Por su parte, la voluntad política cumple un papel determinante, ya que si esta no existe o es muy volátil como en el caso de Venezuela es muy complicado establecer acuerdos que ayuden al beneficio de los países implicados.

No obstante, surgen preguntas interesantes tales como si el objetivo final es la existencia de un único mercado o por el contrario, a pesar de que no haya una convergencia en precios, aún se justifica el funcionamiento de estas transacciones internacionales de electricidad, dado el beneficio que representan no únicamente en términos de la garantía de suministro, sino para los agentes implicados en el proceso, pues es claro que el país con recursos más costosos tiene una reducción en el precio para la prestación del servicio final. 
Es claro, que desde los objetivos de política energética el que prima es el de confiabilidad en el suministro, pues la evidencia del apagón en California en 2005, Brasil en 2008 y el racionamiento en Colombia en 1992 dejan evidencia clara de los altos costos de estos hechos y por lo tanto, así no haya una integración total las TIE traen grandes beneficios en el funcionamiento de los mercados energéticos, ya que permiten utilizar las redes de interconexión para abastecer déficits en periodos adversos.

\section{Referencias}

Barro, R. J. y Sala-i Martin, X. (1992). Convergence. Journal of Political Economy, 100, 223-51.

CAF (2006). Colombia análisis del sector eléctrico. Informes sectoriales de infraestructura, Bogotá: Corporación Andina de Fomento.

Camargo, J. V. (2011). Actas del 12 Encontro Internacional de Energía, Integración Energética: FIESP Avances en la integración electro energética. São Paulo.

Comunidad Andina de Naciones. (2012). Integración eléctrica. Disponible en: http://www.comunidadandina.org/.

CENACE (2010). Informe Anual 2009. Technical report. Quito: Centro Nacional de Control de Energía.

CENACE (2011). Informe Anual 2010. Technical report. Quito: Centro Nacional de Control de Energía.

CENACE (2012). Informe Anual 2011. Technical report. Quito: Centro Nacional de Control de Energía.

CIER (2006). Estudio de las transacciones de electricidad de las regiones Andina, América Central y Mercosur. Factibilidad de su integración. Primera fase. Montevideo: [Informe].

CIER (2011). Sintesis Informativa Energética de los Países de la CIER. Technical report. Montevideo: Comisión de Integración Energética Regional.

CONELEC (2010). Desarrollo de las Transacciones Internacionales de Electricidad en el período de vigencia de la Decisión 720 de la Comunidad Andina. Technical report. Quito: Consejo Nacional de Electricidad.

Congreso Nacional de la República (1996). Ley del Régimen del Sector Eléctrico CONELEC (2008 y 2010), Regulación CONELEC 006 de 2008, Regulación CONELEC 004 de 2010.

Congreso de la República (1994), Ley 142 de 1994, "Ley de Servicios Públicos". Congreso de la República (1994), Ley 143 de 1994, "Ley de Servicios Públicos". CREG (2003, 2004 y 2009), Resoluciones CREG 004 de 2003, CREG 006 de 2003, CREG 014 de 2004 y CREG 160 de 2009. 
Empresa Propietaria de la Red (2012). Operación del mercado eléctrico regional. Disponible en http:/ / www.eprsiepac.com/operacionmersiepactransmisioncostarica.htm.

Evans, L., Guthrie, G. y Videbeck, S. (2008). Assessing the Integration of Electricity Markets Using Principal Component Analysis: Network and Market Structure Effects. Contemporary Economic Policy, 26(1), 145-161.

García, J. J. y Palacios, C. M. (2006). La integración energética de los países nórdicos -nord pool-: lecciones para otros mercados. Lecturas de Economía, 64, 119-142.

García, M. (Ed.) (2006). Sistemas de seguimiento a mercados eléctricos internacionales. Aplicación a países de la Región Andina. Bogotá: Colciencias, XM y CID.

Glanchant, J. M. y Pignon, V. (2003). Nordic Electricity Congestion's Arrangement as a Model for Europe: Physical Constraints or Operators' Opportunity. Cambridge Working Papers in Economics 0313, 1-26.

Sierra, J. y Jaramillo, A. (2007). Aproximación a un análisis económico del comportamiento de los agentes en el modelo de market splitting para las transacciones internacionales de electricidad mediante teoría de juegos y el uso de la conjetura bertrand-edgeworth. Ensayos de Economía, 30, 91-104.

Wolszczak-Derlacz, J. (2008). Price Convergence in the eu-an Aggregate and Disaggregate Approach. International Economics and Economic Policy, 5, 2547. Doi: $10.1007 /$ s10368-008-0104-1.

XM (2012a). Descripción del sistema eléctrico colombiano. Disponible en http:/ / www.xm.com.co/pages/descripciondelsistemaelectricocolombiano.aspx.

XM, Ed. (2012b). Despacho Económico, Planeación, Programación y Coordinación de la operación. Medellín: XM.

Zachmann, G. (2008). Electricity Wholesale Market Prices in Europe: Convergence? Energy Economics, 30(4), 1659-1671. 
